\title{
List of Lectures by Speakers Who Have Not Submitted Their Manuscripts
}

P-Selectin Expression in Human Dermal Microvascular Endothelial Cells

Yasuhiro Miyazaki et al.

Department of Dermatology and Immunodermatology, Graduate School, Tokyo Medical and Dental University, Tokyo, Japan

\section{Augmentation of Allergic Inflammation by Formaldehyde Exposure - Analysis Using an Antigen-Specific Murine Asthmatic Model Hirofumi Ishida et al. \\ Department of Internal Medicine, Teikyo University, School of Medicine, Tokyo, Japan}

\section{LTD $_{4}$ Induces Eosinophil Degranulation and Superoxide Production via $\boldsymbol{\beta}_{\mathbf{2}}$ Integrin \\ Keiko Saito et al. \\ Department of Respiratory Medicine, Saitama Medical School, Saitama, Japan}

\section{Effects of CpG ODN on Human Eosinophils}

Kenji Matsumoto et al.

Department of Allergy and Immunology, National Center for Child Health and Development Research Institute, Tokyo, Japan
Expression and Regulation of Toll-Like Receptor 2 in Cord Blood-Derived and Adult Peripheral Blood-Derived Cultured Human Mast Cells

Naoko Inomata et al.

Department of Dermatology, Yokohama City University of Medicine, Yokohama, Japan

Expression and Function of Toll-Like Receptors in Eosinophils - Activation by Toll-Like Receptor 7 Ligand

Hiroyuki Nagase et al.

Departments of Respiratory Medicine, University of Tokyo Graduate School of Medicine, Tokyo, Japan

Characteristics of Cells Gathered in Effusion of the Middle Ear in a Patient with Eosinophilic Otitis Media

Akiko Tokumaru et al.

Department of Otolaryngology and Head and Neck Surgery, Koshigaya Hospital, Dokkyo University School of Medicine, Saitama, Japan

\section{KARGER \\ (C) 2004 S. Karger AG, Basel}

Fax +4161306 1234 